\title{
HTS Motor Performance Evaluation by Different Pulsed Field Magnetization Strategies
}

\author{
Zhen Huang, H. S. Ruiz, Wei Wang, Zhijian Jin, and T. A. Coombs
}

\begin{abstract}
A fully high temperature superconducting (HTS) motor has been built in the EPEC laboratory at the University of Cambridge, UK. Two different pulsed field magnetization (PFM) strategies have been implemented for studying the magnetization of a cylindrical rotor designed by an asymmetric array of superconducting bulks. Asymmetric or uneven distribution of superconducting bulks could occur when the size of the superconducting bulks is compromised due to the curvature of the rotor and the design aims to cover the largest area as possible for the magnetic poles. It is certainly an unusual design which nevertheless deserve to be explored, as possible misalignments between the superconducting poles to be magnetized by PFM can be studied when an uneven arrangement of columns of superconducting bulks is implemented for along a cylindrical shaft. In the first of the PFM strategies, only six out of fifteen columns of superconducting bulks have been effectively magnetized, and the performance of the resulting rotor has been compared with the case when the PFM strategy leads to the magnetization of up to $93 \%$ of the superconducting bulks. The advantages and disadvantages of each of the PFM strategies is discussed.
\end{abstract}

Index Terms - fully HTS synchronous motor, HTS rotor, pulsed field magnetization

\section{INTRODUCTION}

$\mathrm{H}$ igh temperature superconducting machines employing state of the art type-II superconducting (SC) bulks and the second generation of high temperature superconducting (2G-HTS) wires, are all expected to come to the phase of commercialization in the next five to ten years if a continuous reduction of prices of SC materials is kept. Nevertheless, more studies are still required to understand the complex magnetic response of SC materials in rotary machines like motors and generators, where a strong reduction on the total weight of the machine together with a significant increase on its rated power, are both expected by the replacement of the copper windings and/or permanent neodymium-based magnets by SC materials [1]. Some of these technologies have been demonstrated in the past by different groups either by using Gd-based superconducting bulks on the designing of an axial-gap type synchronous propulsion motor [2], or by using 2G-HTS wires for the prototyping of synchronous induction motors [3]. Likewise, at the Department of Engineering of the University of Cambridge, U.K., the concept of a fully HTS

Manuscript received September 03, 2016.

Zhen Huang, Zhijian Jin are with the Academy of Information Technology and Electrical Engineering, Shanghai Jiao Tong University, Shanghai 200240, China (e-mail: zhen.huang@sjtu.edu.cn).

H. S. Ruiz is with the Department of Engineering, University of Leicester, Leicester, Leicester LE1 7RH, UK.

Wei Wang is with the School of Electrical Engineering and Information, Sichuan University, Chengdu 610065, China.

T. A. Coombs is with the Division of Electrical Engineering, Department of Engineering, University of Cambridge, Cambridge CB3 OFA, UK.

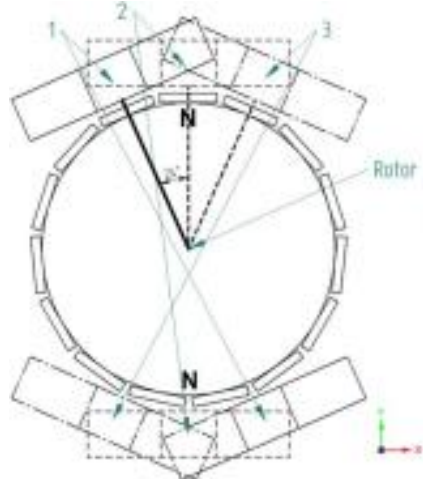

(a)

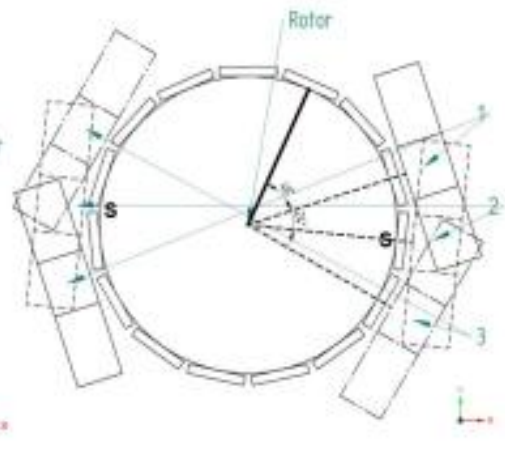

(b)
Fig. 1. The extended pulsed field magnetization strategy is designed to produce a pair of (a) $\mathrm{N}$ poles and (b) $\mathrm{S}$ poles with larger pole areas. 1, 2, and 3 are the three pulse positions (starting from 1).

motor has been studied since 2005 [4], where multiple stator and rotor designs have been explored [5-10]. In the original design [4], the field and armature windings of the EPEC HTS motor were intended to be made of 2G-HTS wires, but this idea was rapidly abandoned due to the high cost of the machine and the lack of knowledge on the performance of stacks of superconducting wires subjected to crossed and rotating magnetic fields, the latter an issue recently covered by some of the authors of this paper [11]. Thus, the four-pole motor design had to be reconsidered in 2007 [5], leading to the prototyping of the first fully HTS superconducting motor at EPEC, with the rotor being composed by an array of seventy-five YBCO superconducting bulks [12] attached to the surface of a cylindrical shaft [6], and the stator is made of an array of six 2G-HTS racetrack coils [7]. However, the control and operation of this motor in synchronous regime demonstrated to be too challenging at this stage, and it was not up to 2013 when an adequate control system was embedded [8]. This system, which in brief is a variable-voltage variable-frequency open-loop control system, paved the way in 2014 for the realization of the first tests with a continuous operation of the HTS motor in a liquid nitrogen bath [9]. For a more thorough discussion on the experimental setup and the devised HTS motor at the EPEC laboratories, we recommend to the reader to see references [6-9]. Here, it is only necessary to notice that the magnetic poles of the rotor configuration in [9] where created by the PFM of individual columns of five HTS bulks, and this strategy was studied in detail in [10]. In fact, in this case only 4 out of up to 15 columns of HTS bulks have been used, what gives room to magnetize a larger area per pole if a greater number of columns is subjected to the PFM. Thus, in this paper we focus on the possibility to magnetize an array of multiple columns of HTS bulks for conforming the magnetic poles of the EPEC HTS motor, and the effects of having an asymmetric distribution of bulks as result of an uneven number of columns.

This paper is organized as follows: In Section II, the new PFM strategy for the magnetization of multiple columns of HTS bulks 


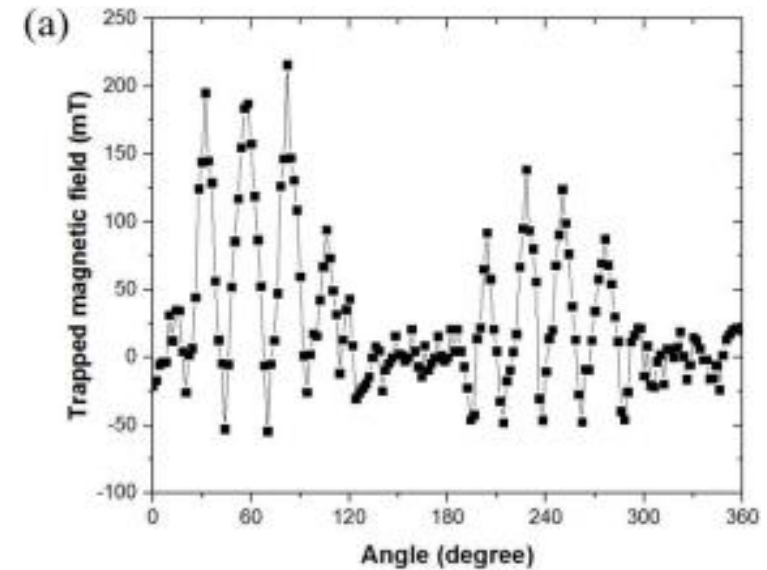

(b)

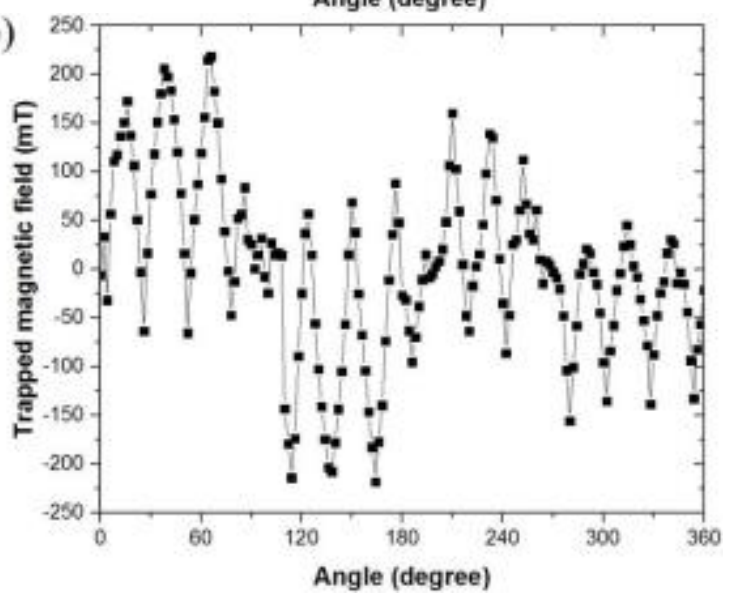

Fig. 2. (a) $360^{\circ}$ field profile measured $4 \mathrm{~mm}$ away from the rotor surface after nine magnetization pulses. (b) same as above, but after the eighteen pulses procedure, i.e., three pulses are initially applied to produce the N-poles, and then another three pulses are applied to produce the S-poles of the rotor.

is described. Then, in Section III the equivalent circuit parameters of the EPEC HTS motor and its performance is investigated under the different PFM approaches. Finally, in Section IV a summary of the main conclusions derived from this study is presented.

\section{The EXTENDED PULSED FIELD MAGNETIZATION STRATEGY}

For the sake of discussion and brevity of this manuscript, the results presented below are limited to the description of the socalled extended PFM strategy for the EPEC HTS motor, where multiple columns of HTS bulks (per pole) have been magnetized. Nevertheless, for a comprehensive description of the original PFM strategy which implies the magnetization of poles made of singular columns of HTS bulks, we encourage the reader to read Ref. [10]. In simple teRMS, the extended PFM strategy is designed to produce a couple of pairs of $\mathrm{N}$ - and S-poles with larger magnetized areas than the ones obtained by the original PFM strategy, which eventually could lead to a significant increase on the efficiency figures for the EPEC HTS motor.

The extended PFM strategy is as follows: the angular position of the rotor has been carefully adjusted such that the wider area of one of the magnetization coils (see Fig. 3 in Ref. [10]) faces the wider surface of one of the columns of HTS bulks which is to be magnetized (see Fig. 1 in Ref. [10]). This position must be mechanically clamped to counteract the possible misalignment of the rotor and the magnetization coils, it due to the magnetic torque induced by the pulsed magnetic field. In a two-dimensional view of the system, it being seen from the top of the rotor with circular cross-section, the new PFM strategy can be illustrated in Fig. 1. It is to be noticed that three different positions for the magnetization coils are shown in Fig. 1 (a), each referring for the magnetization of an individual column of HTS bulks. Thus, for the first position, three pulses of $1.5 \mathrm{~T}$ (measured at the centre of the magnetizing coil) are applied within intervals of $5 \mathrm{~min}$, allowing for the magnetic relaxation of the system and the charging period of the bank of capacitors connected to the magnetization coils. The characteristics of the magnetization coils under different charging voltages can be found in Ref. [10]. Then, for the magnetization of the second and third column of HTS bulks, rotations of 24 degrees each are applied, and the procedure above described is repeated at these positions (see Fig. 1 (a)), resulting hence a pair of S-poles whose area covers at least three columns of HTS bulks. Likewise, the pair of N-poles are created by following the same PFM strategy but inverting the charging direction of the bank of capacitors, and rotating further 48 degrees for the magnetization of the first column of HTS bulks inside the new N-pole (see Fig. 1 (b)).

For understanding the experimental results, it is to be noticed in Fig. (1) that the relative alignment between the magnetization coils and the columns of HTS bulks that conform each one of the pair of S- and N-poles, does not always show the face of the magnetization coil straightforwardly aligned with the face of a column of HTS bulks. In fact, it can be seen that for the magnetization coil displayed at the bottom of Fig. 1 (a), the centre axis of this magnetization coil is in fact aligned with the intersection between two successive columns of bulks. Therefore, whilst the upper coil aims to magnetize a single column of HTS bulks, the coil displayed at the bottom of Fig. 1 (a) aims to magnetize two columns of HTS bulks with their wider surface not orthogonally oriented with the direction of the applied magnetic field. Thus, although the allocation of an even number of columns of HTS bulks is preferable, the current distribution of HTS bulks allows exploring simultaneously two different arrangements of HTS bulks for the generation of poles with wider magnetized areas, by applying the same number of magnetic pulses. Naturally, the main drawback of this design is the need to redesign the original variable-voltage variable-frequency open-loop control system [8], which is to be published elsewhere. However, in order to reformulate this control system is first necessary to measure the magnetization profiles of the new rotor, being this the main aim of this paper.

Thus, by measuring the magnitude of the trapped magnetic field along the radial direction at the middle height of the array of columns of HTS bulks (Fig. 2), it is possible to observe that the first of the N-poles (Fig. 2 (a)), and the first of the S-poles (Fig. 2 (b)), are both conformed by an array of three columns of magnetized HTS bulks, whilst the second of the N- and S-poles is composed by an array of up to four columns of magnetized HTS bulks, as result of the relative orientation between the magnetization coils and the columns of superconducting bulks displayed in Fig. 1. Similar results have been obtained at the centre of each one of the five HTS bulks that conform the individual columns displayed in Fig. 1. However, it is to be noticed that the averaged peaks of trapped magnetic field for the pairs of N-S poles differs when the relative orientation between the magnetization coils and the superconducting bulks is different, as it was explained before. Therefore, it is not surprising that for the same intensity of applied magnetic field, the amount of trapped field becomes lower for those superconducting bulks with their wider surface not perfectly aligned with the magnetization coil, although in return a greater magnetized area is achieved. 


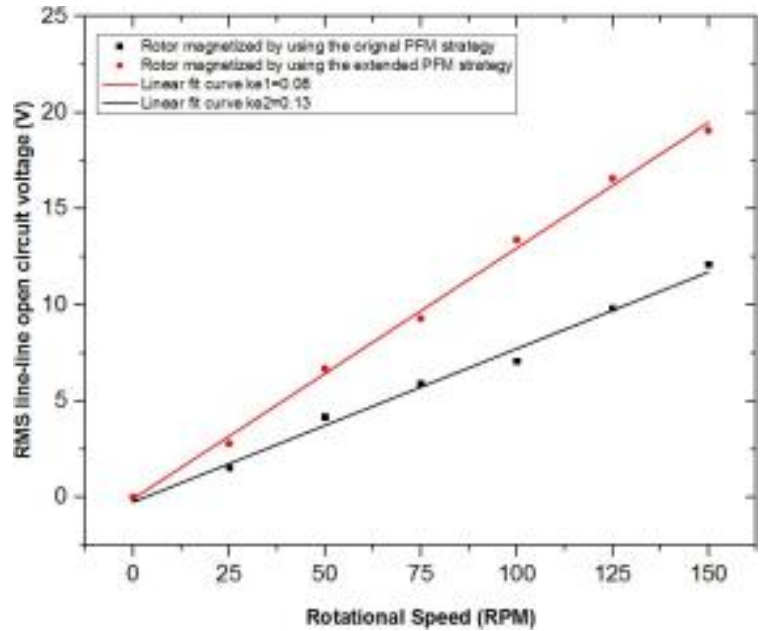

Fig. 3. Line-line (RMS) open circuit voltage versus rotational speed.

\section{SuPERCONDUCTING Motor PERFORMANCE EVALUATION AND EQUIVALENT CIRCUIT PARAMETERS ESTIMATION}

The fully high temperature superconducting motor designed in the EPEC laboratory is aimed to operate as a four non-salient poles' HTS synchronous motor. A simplified analysis of its performance can be pursued when the HTS motor reaches a steady state, then being possible to calculate the fundamental parameters of operation of the motor by the one phase steady-state equivalent circuit of a synchronous motor. Thus, the one phase steady-state equation can be written as,

$$
U=E_{0}+R_{s} I_{a}+j X_{s} I_{a}
$$

where $E_{0}$ is the excitation voltage, $U$ is the terminal phase voltage, $I_{a}$ is the stator winding current, $R_{s}$ is the equivalent resistance of $2 \mathrm{G}$ HTS coils on the stator, and $X_{s}$ is the synchronous reactance.

An open-loop voltage/frequency drive system has been designed for achieving the steady-state operation of the HTS motor, then allowing us to control its speed by balancing the sinusoidal magnetic motive forces in the airgap between the stator and the rotor [8]. Thus, during this test and after implementing the original PFM strategy described in Ref. [10], the motor has been accelerated with a rate of $0.1 \mathrm{~Hz} / \mathrm{s}$ up to reaching $150 \mathrm{rpm}$, and with a light load (ZHEN: What do you mean with light load? you must specify this as a percentage of the rated load because otherwise the calculation and values of efficiency lack of meaning). Then, the measured peak voltage at $150 \mathrm{rpm}$ is about $24.5 \mathrm{~V}$ (in RMS units), which is limited by our power source. During the running time of the HTS motor, an AC/DC clamp meter is used to measure the RMS value of phase current (26 A), which is $80 \%$ of the measured RMS critical current $(32.5 \mathrm{~A})$ of the superconducting coils used in our motor [9]. Therefore, to protect the superconducting coils we may assume that the rated RMS values for the line current $\left(I_{l 1}\right)$, line voltage $\left(U_{l 1}\right)$, and angular speed $\left(\omega_{r l}\right)$ are $26 \mathrm{~A}, 24.5 \mathrm{~V}$, and $150 \mathrm{rpm}$ respectively, when the rotor of the HTS motor has been magnetized by the original PFM strategy.

Likewise, for the extended PFM strategy, we have determined that the RMS rated values of the motor are $I_{l 2}=18 \mathrm{~A}, U_{l 2}=24.5$ $\mathrm{V}$, and $\omega_{r 2}=150 \mathrm{rpm}$. Thus, with this new design of the rotor, we have calculated the motor voltage constant $\left(k_{e}\right)$ by measuring the

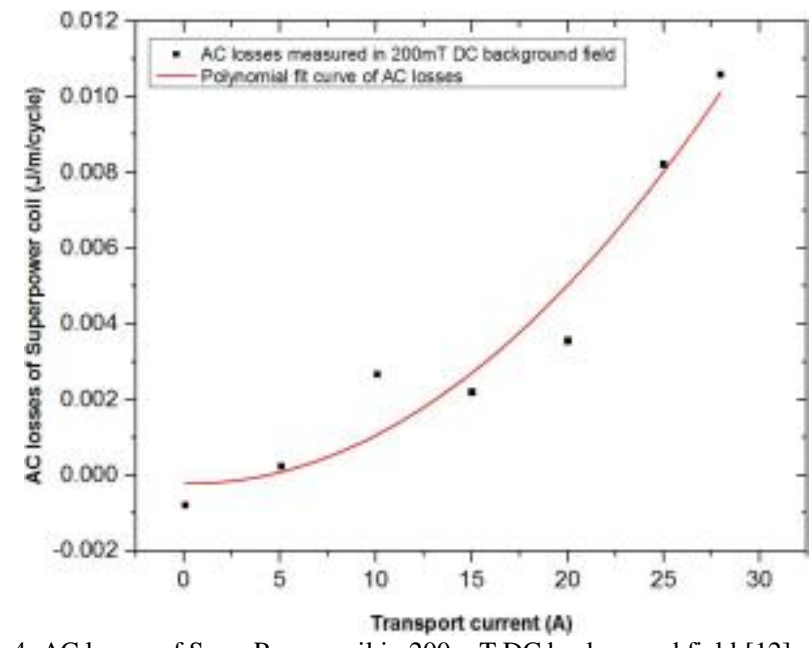

Fig. 4. AC losses of SuperPower coil in $200 \mathrm{mT}$ DC background field [12].

line-to-line voltage across two phases of the HTS motor under open circuit conditions and for various rotational speed. In our experiments, the rotational speed varies from $0 \mathrm{rpm}$ to $150 \mathrm{rpm}$ (i.e., $2.5 \mathrm{~Hz}$ ).

Figure 3 shows the RMS line-to-line open circuit voltage as a function of the rotational speed, from which it is possible to calculate the voltage constant $k_{e}$ (the slope of the curves) for each one of the PFM strategies, i.e, for the original $\left(k_{e l}=0.08\right)$ and extended $\left(k_{e 2}=0.13\right)$ PFM strategies. On the one hand, it is to be noticed that the increment of voltage constant of the motor after the extended PFM strategy, $k_{e 2}$, is a consequence of the increase on the area of the magnetized poles, which now consider a greater number of columns of HTS bulks (Fig. 2 (b)). On the other hand, the equivalent resistance $R_{s}$ to be considered in the one-phase equivalent circuit can be calculated by the principle of conservation of energy,

$$
Q f=I_{r m s}^{2}\left(R_{s} / 2\right),
$$

where $Q$ reads for the AC losses of the 2G HTS coil, $f$ is for the frequency of the transport current across the coil, and $I_{\mathrm{RMS}}$ is its rated current. Nevertheless, due to the size of the motor which overcomes the dimensions of our facilities for the experimental measurement of AC losses by the calorimetric method, in a first approach $Q$ has been estimated from the experimental measurement of the AC losses in a single 2G HTS coil (Figure. 4), discussed in Ref. [13]. In particular, the results displayed in Fig. 4 correspond to the measured $\mathrm{AC}$ losses of a coil of the same dimensions than the coils used in our motor, but subjected to a perpendicular ripple magnetic field of $200 \mathrm{mT}$ (at $10 \mathrm{~Hz}$ ) which is about the maximum trapped magnetic field observed in Fig. 2. Being this the closest experimental data available in the literature, a polynomial fit of the curve displayed in Fig. 4 reveals than the expected AC losses under the original and extended PFM approaches are about $Q_{1}=2.16 \mathrm{~J}(0.0087 \mathrm{~J} / \mathrm{cycle} / \mathrm{m} \times 5$ cycle $\times$ $49.7 \mathrm{~m})$ and $Q_{2}=0.994 \mathrm{~J}(0.004 \mathrm{~J} /$ cycle $/ \mathrm{m} \times 5$ cycle $\times 49.7 \mathrm{~m})$, respectively, being $49.7 \mathrm{~m}$ the total length of the $2 \mathrm{G}$ HTS tape (Superpower 344) used for the fabrication of the coil. Thus, at $150 \mathrm{rpm}$, i.e., for $f=2.5 \mathrm{~Hz}$, the equivalent resistance $R_{s}$ for both PFM strategies accounting for the set of two coils per pole, results:

$$
R_{S 1}=\frac{4 Q_{1}}{I_{l 1}^{2}} f \approx 6.39 \times 10^{-3} f=32 \mathrm{~m} \Omega
$$




$$
R_{S 2}=\frac{4 Q_{2}}{I_{l 2}^{2}} f \approx 6.14 \times 10^{-3} f=30.7 \mathrm{~m} \Omega,
$$

Then, from Eq. (3.1) the synchronous inductance $\left(L_{s}\right)$ can be evaluated by,

$$
L_{S}=\sqrt{\frac{U^{2}-E_{0}^{2}}{\left(2 \pi f I_{a}\right)^{2}}},
$$

which for the open circuit voltages displayed in Fig. 3 (at $125 \mathrm{rpm}$ ), and a measured terminal phase voltage of $14.14 \mathrm{~V}$, results in $L_{s 1}=15.1 \mathrm{mH}$ for the original PFM strategy case, and $L_{s 2}=15 \mathrm{mH}$ for the extended PFM strategy. Finally, to estimate the efficiency of the HTS motor, in a first approach, we have measured the rotor torque by a Honeywell FS series force sensor with an embedded Wheatstone bridge circuit. When measuring the torque, the rotor is locked, and the phase current in the stator windings is increased from $0 \mathrm{~A}$ up to $5 \mathrm{~A}$. The transport current is adjusted by three rheostats which are connected to each phase of the stator windings, and the current is monitored by an AC/DC clamp meter.

Figure 5 shows the torque versus the RMS phase current of the motor, from which the torque constant, $k_{t}$, for each of the PFM strategies can be calculated by the slope of the fitting curves. Then, for the original PFM strategy we have determined that $k_{t 1}=1.23$, whilst for the extended PFM strategy a greater torque constant is obtained, $k_{t 1}=1.72$, as consequence of the greater size of the magnetized areas over each of the four poles of the motor. The ratios between the torque constants and voltage constants for both cases are $k_{t 1} / k_{e 1}=15.4$ and $k_{t 2} / k_{e 2}=13.2$, respectively, both close enough to the analytical result $\sqrt{3} \times 60 / 2 \pi \times k_{e}=16.5$. Thus, for the corresponding RMS phase currents $I_{t 1}=26 \mathrm{~A}$, and $I_{t 2}=18 \mathrm{~A}$, the rated torques for the original and extended PFM strategies result to be $\Gamma_{1}=31.98 \mathrm{Nm}$ and be $\Gamma_{2}=30.96 \mathrm{Nm}$, respectively.

Therefore, the input power for each strategy results to be:

$$
\begin{aligned}
& P_{i n 1}=\sqrt{3} U_{l 1} I_{l 1} \cos \phi \approx 882.7 \mathrm{~W}, \\
& P_{i n 2}=\sqrt{3} U_{l 2} I_{l 2} \cos \phi \approx 611.1 \mathrm{~W},
\end{aligned}
$$

where $P_{i n 1}$ is the rated input power of the HTS motor when the rotor has been magnetized by using the original PFM strategy, and $P_{i n 2}$ is the rated input power of the HTS motor when the rotor has been magnetized by using the extended PFM strategy. Then, from the values obtained from the rated torques, the output power for each of these cases is:

$$
\begin{aligned}
& P_{\text {out } 1}=T_{1} \omega_{r} \approx 502.7 \mathrm{~W}, \\
& P_{\text {out } 2}=T_{2} \omega_{r} \approx 486.9 \mathrm{~W},
\end{aligned}
$$

Thus, for the XXX \% load of our experiments, the efficiencies of the EPEC HTS motor under the original and extended PFM strategies at $125 \mathrm{rpm}$ are:

$$
\begin{gathered}
\eta_{1}=\frac{P_{\text {out } 1}}{P_{\text {in } 1}} \times 100 \%=57 \% \\
\eta_{2}=\frac{P_{\text {out } 2}}{P_{\text {in } 2}} \times 100 \%=80 \%
\end{gathered}
$$

It is worth mentioning that for a conventional nonsuperconducting four phase motor, the efficiency decreases dramatically under the minimum efficiency for acceptable operation (50\%) with loads lesser than 50\% of the rated load [14]. Therefore, not only a $23 \%$ increment on the efficiency of the HTS

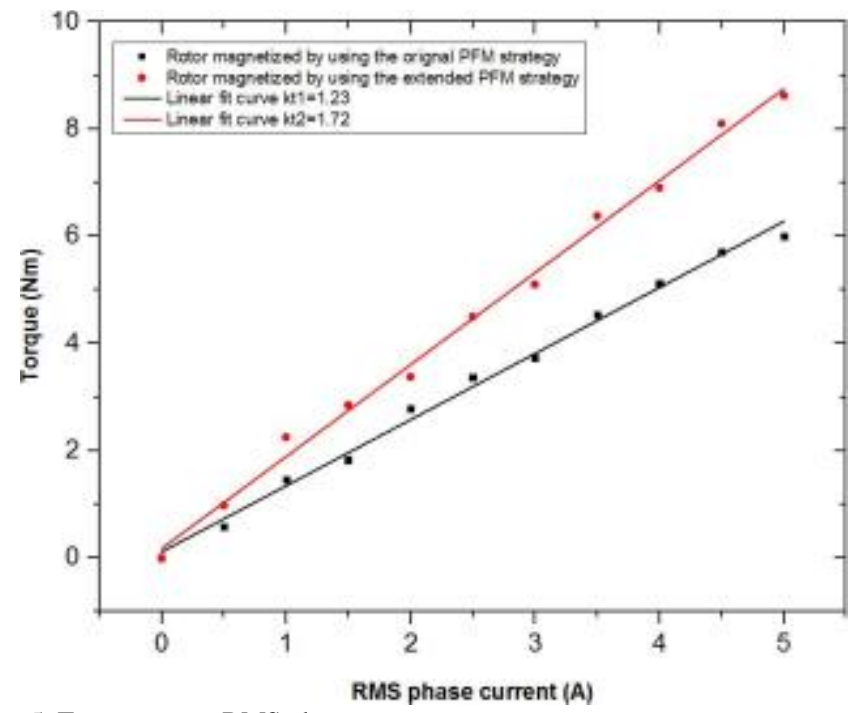

Fig. 5. Torque versus RMS phase current.

motor has been achieved under the extended PFM strategy but also, we have demonstrated the superior features of the HTS motor over conventional non-superconducting designs, although significant complexity is added in terms of the voltage/frequency and speed control systems, besides the need of designing an adequate cryogenic environment.

\section{CONCLUSION}

In this paper a new PFM strategy for the magnetization of multiple columns of HTS bulks in the so-called EPEC HTS motor is presented. This strategy extends the original PFM strategy presented in Ref. [10], and introduce the experimental evaluation of the fundamental criteria for determining the efficiency of the motor under the approach of one phase steady-state equivalent circuit. The results indicate that the efficiency of the EPEC HTS motor can increase in at least a $23 \%$ by the extended PFM strategy, when the amplitude of the magnetic field applied during the original PFM strategy is conserved. The main difference between the original and extended PFM strategies can be observed in the diagrams shown in Fig. 1, where the original PFM strategy reads for the magnetization of a single column of HTS bulks per pole, rather than the successive magnetization of three columns of HTS bulks for the extended PFM strategy. Thus, despite an uneven number of columns of HTS bulks has been implemented in this design, it has been proven that the motor can operate in steadystate conditions, although the designing of the voltage/frequency and speed control systems offers additional challenges. Nevertheless, by taking benefit of the experience acquired with the EPEC HTS motor, and the fact that reinforced superconducting bulks have been proven to be capable to trap up to $17.6 \mathrm{~T}$ [15], it is possible to envisage the optimal designing of a fully superconducting motor for high power applications in the future, following the principle of a cylindrical magnetized rotor composed by columns of HTS bulks.

\section{REFERENCES}

[1] A. M. Campbell, "A superconducting magnetic gear," Superconductor Science and Technology, vol. 29, no. 5, pp. 054008, 2016.

[2] M. Miki, S. Tokura, and H. Hayakawa et al., "Development of a synchronous motor with $\mathrm{Gd}-\mathrm{Ba}-\mathrm{Cu}-\mathrm{O}$ bulk superconductors as pole-field magnets for propulsion system," Superconductor Science and Technology, vol. 19, no. 7 pp. S494, 2006.

[3] K. Nagao, T. Nakamura, and T. Nishimura et al., "Development and fundamental characteristics of a YBCO superconducting 
induction/synchronous motor operated in liquid nitrogen," Superconductor Science and Technology, vol. 21, no. 1, pp. 015022, 2008.

[4] Q. Jiang, M. Majoros, Z. Hong, A. Campbell, and T. Coombs, "Design and ac loss analysis of a superconducting synchronous motor," Superconductor Science and Technology, vol. 19, no. 11, pp. 1164, 2006.

[5] Y. Jiang, R. Pei, Z. Hong, J. Song, F. Fang, and T. Coombs, "Design and control of a superconducting permanent magnet synchronous motor," Superconductor Science and Technology, vol. 20, no. 7, pp. 585, 2007.

[6] Y. Jiang, R. Pei, Z. Hong, Q. Jiang, and T. Coombs, "Design of an hts motor," in Journal of Physics: Conference Series, vol. 97, pp. 012123, IOP Publishing, 2008.

[7] Y. Jiang, R. Pei, W. Xian, Z. Hong, and T. Coombs, "The design, magnetization and control of a superconducting permanent magnet synchronous motor," Superconductor Science and Technology, vol. 21, no. 6, pp. 065011, 2008.

[8] Z. Huang, W. Xian, and M. Zhang, et al., "Control and operation of a high temperature superconducting synchronous motor," IEEE Transactions on Applied Superconductivity, vol. 23, no. 3, pp. 5200204-5200204, 2013.

[9] Z. Huang, M. Zhang, W. Wang, and T. A. Coombs, "Trial test of a bulk-type fully HTS synchronous motor," IEEE Transactions on Applied Superconductivity, vol. 24, no. 3, pp. 1-5, 2014.

[10] Z. Huang, H. S. Ruiz, and Yujia Zhai, et al., "Study of the pulsed field magnetization strategy for the superconducting rotor," IEEE Transactions on Applied Superconductivity, vol. 26, no. 4, pp. 5202105, 2016.

[11] M. Baghdadi, H. S. Ruiz, and T. A. Coombs, "Crossed-magnetic-field experiments on stacked second generation superconducting tapes: Reduction of the demagnetization effects," Applied Physics Letters, vol. 104, pp. 232602, 2014.

[12] V. Plechacek, M. Jirsa, M. Rames, and M. Muralidhar, "Batch production of YBCO disks for levitation applications," Physics Procedia, vol. 36, pp. 538543,2012

[13] M. Chudy, Y. Chen, and M. Zhang, et al., "Power losses of 2g hts coils measured in external magnetic dc and ripple fields," IEEE Transactions on Applied Superconductivity, vol. 24, no. 1, pp. 111-116, 2014.

[14] H. E. Jordan, "Energy-Efficient Electric Motors and their Applications," published by Springer US, isbn 9781489914675, 2013.

[15] J. H. Durrell, A. R. Dennis, and J. Jaroszynski, et al., "A trapped field of 17.6t in melt-processed, bulk gd-ba-cu-o reinforced with shrink-fit steel," Superconductor Science and Technology, vol. 27, no. 8, pp. 082001, 2014. 